\title{
Transition from open to robotic-assisted radical prostatectomy is associated with a reduction of positive surgical margins amongst private-practice-based urologists
}

\author{
Ralph Madeb • Dragan Golijanin • Joy Knopf • \\ Craig Nicholson • Stuart Cramer · Frederick Tonetti • \\ Kelly Piccone • John R. Valvo • Louis Eichel
}

Received: 8 December 2006 / Accepted: 15 January 2007 / Published online: 23 February 2007

(C) Springer London 2007

\begin{abstract}
Several recent studies have suggested that thought leaders in radical prostatectomy have decreased their own positive margin rates by switching from open to robot-assisted radical prostatectomy. Theoretically, this improvement is largely attributed to enhanced visualization of the deep pelvis and precision of dissection afforded by the instrumentation. To date, it has not been determined if this phenomenon exists amongst non-fellowship-trained urologists in private practice. Herein, we describe the positive margin rates of two non-fellowship-trained private-practice urologists who converted from open radical retropubic prostatectomy to robot-assisted radical prostatectomy. The margin positivity data from two non-fellowshiptrained private-practice urologists (surgeon 1 and surgeon 2) were reviewed retrospectively. The last 50 cases of open radical retropubic prostatectomy from each surgeon were compared with the first 50 robotic prostatectomy cases of surgeons 1 and 2, respectively. A positive surgical margin was defined as tumor present at the inked margin of the prostate. There was a significant decrease in the overall and pT2 positive margin rates for both surgeons. The overall positive margin rate and $\mathrm{pT} 2$ positive margin rate for surgeon 1 dropped from 44 to $20 \%$ and from 37 to $5.7 \%$, respectively, after changing from open to robotic prostatectomy. For surgeon 2, the overall positive margin rate
\end{abstract}

R. Madeb $(\bowtie) \cdot$ D. Golijanin · J. Knopf · C. Nicholson · S. Cramer $\cdot$ F. Tonetti $\cdot$ K. Piccone $\cdot$ J. R. Valvo $\cdot$ L. Eichel Departments of Urology and Pathology,

Polisseni Robotic and Minimally Invasive Surgery Center, Rochester General Hospital, 2615 Culver Road,

Rochester, NY 14609, USA

e-mail: rrm@rochester.rr.com changed from 26 to $18 \%$ and the pT2 positive margin rate changed from 27.5 to $7 \%$ after converting. Changing from open to robotic-assisted radical prostatectomy may improve the ability of urologists to obtain negative surgical margins. With proper training this phenomenon does seem to apply to non-fellowship-trained urologists in private practice and can be realized within the first 50 cases performed.

Keywords Radical prostatectomy - Prostate cancer · Robotic-assisted $\cdot$ Positive margins

\section{Introduction}

An ever-increasing number of prostatectomies are being performed using the da Vinci robotic system [14]. Multiple reports over the past five years have described the advantages that are characteristically seen with robotic surgery, including decreased blood loss, shorter hospital stay, and a shorter convalescence with a faster return to daily activities and work [5-10]. More recently, functional results with regard to potency and continence after robotic prostatectomy have also been reported and have demonstrated results that are equal to the open approach [11-16].

In addition to issues regarding expedited recovery and potential advantages with regard to urinary control and sparing of potency, the primary goal of cancer control still remains the primary goal of the operation [17]. For robotic prostatectomy to be considered an alternative to the open approach, cancer control must be equivalent or better [17-20]. Because robotic prostatectomy is a relatively new technique, long-term follow-up data regarding cancer control is not available. 
An early marker for oncologic efficacy after radical prostatectomy is the positive surgical margin rate. Cancer at the inked margin suggests incomplete resection and may be associated with a worse prognosis. Although, this point can be debated, a growing body of investigators agree that a positive margin may adversely affect cancer control [17-27]. Several recent studies have suggested that leaders in robotic prostatectomy have decreased their own positive margin rates by switching from open to robotic radical prostatectomy [5, 28-31]. Theoretically, this improvement is largely attributed to enhanced visualization of the deep pelvis and precision of dissection afforded by the instrumentation. To date, it has not been determined if this phenomenon is transferable to non-fellowship trained urologists in private practice. Herein, we describe the positive margin rates of two non-fellowship trained private practice urologists who converted from open radical retropubic prostatectomy to robotassisted laparoscopic radical prostatectomy.

\section{Methods}

Patients and study design

Our study was a retrospective outcome analysis of radical prostatectomies performed by two non-fellowshiptrained private-practice urologists. Both surgeons (J.R.V. and F.W.T.) have performed over 1,000 prostatectomies over a 20-year period. Their method of choice until September 2004 for removal of the prostate for clinically localized prostate cancer was open radical retropubic prostatectomy. In September 2004 both surgeons changed to robotic prostatectomy for all radical prostatectomies. Operative and pathologic data was collected via retrospective chart review for the last 50 open radical prostatectomies performed by surgeons 1 and 2 and compared to similar data collected for the first 50 robotic prostatectomies. Table $1 \mathrm{com}-$ pares the demographics of both cohorts.

The indications for robotic and open prostatectomy were identical. Patients with clinically localized prostate cancer were offered all standard treatment modalities including watchful waiting, hormonal ablation therapy, radical prostatectomy, and radiotherapy (brachytherapy and external beam). Previous abdominal surgery, including preperitoneal hernia repair with mesh, was not considered to be a contraindication for robotic prostatectomy. Open radical prostatectomy was performed as described by Walsh et al. [32, 33], while the robotic approach was performed as described by Ahlering et al. [5, 28]
Table 1 Characteristics of both surgeons' last 50 open retropubic and first 50 robotic prostatectomies

\begin{tabular}{llll}
\hline Characteristic & $\begin{array}{l}\text { Open } \\
\text { cases }\end{array}$ & $\begin{array}{l}\text { Robotic } \\
\text { cases }\end{array}$ & $P$ value \\
\hline $\begin{array}{l}\text { Number of cases } \\
\text { (both surgeons) }\end{array}$ & 100 & 100 & \\
$\begin{array}{l}\text { Age } \\
\text { Pre-operative }\end{array}$ & 64.9 & 62.6 & 0.03 \\
$\quad$ PSA (ng/ml) & 8.51 & 7.33 & 0.3 \\
$\begin{array}{l}\text { Gleason score } \\
\text { (median) }\end{array}$ & 6 & 6 & 0.4 \\
$\begin{array}{l}\text { Mean prostate } \\
\text { weight }\end{array}$ & 51.0 & 42.3 & 0.01 \\
$\begin{array}{l}\text { EBL (mean) (cc) } \\
\text { Low-risk profile } \\
\text { Intermediate-risk } \\
\text { profile }\end{array}$ & 710 & 170 & 0.001 \\
$\begin{array}{l}\text { High-risk profile } \\
\text { c }\end{array}$ & 62 & 59 & 0.8 \\
\end{tabular}

\footnotetext{
${ }^{\text {a }}$ Low-risk profile $=$ PSA $<10$, Gleason score $\leq 6$, clinical stage $\mathrm{T} 1 \mathrm{c}$ or T2A

b Intermediate-risk profile $=$ PSA 10-20, Gleason score 7, stage $\mathrm{T} 2 \mathrm{~B}$

${ }^{\mathrm{c}}$ High-risk profile $=\mathrm{PSA}>10$, Gleason score $\geq 8$, stage T2C
}

Pathologic data

A positive surgical margin was defined as the presence of tumor at the inked margin of the specimen. The same pathologic teams evaluated the specimens and were aware of the switch from the open to robotic technique. The final first read of the specimen were used for evaluating positive surgical margins. No specimen required a re-read and none of the specimens were changed from their first final read. In addition, there was no change in the method of sectioning or reporting methods during the study period.

Data collection and statistical analysis

All data as outlined in Tables 1 and 2 were collected retrospectively from patient charts. All comparisons were made using the paired $t$ test, with $P<0.05$ considered statistically significant.

\section{Results}

The transition to robotic prostatectomy in our private-practice setting occurred on September 1, 2004. None of the robotic prostatectomies for either surgeon required conversion to open surgery. Since there was a transition point for a change in the surgical technique we do not feel that there is a selection bias between both groups as they represent all new- 
Table 2 Positive surgical margin status by stage for both surgeons performed by the open retropubic and robotic approach

\begin{tabular}{lll}
\hline & Open & Robotic \\
\hline Surgeon 1 & & \\
Mean GG & 6.6 & 6.8 \\
Mean pre-op PSA & 8.1 & 8.3 \\
Mean prostate weight & 49.4 & 40.4 \\
pT2 (all) + margins & 15 of $41(36.6 \%)$ & 2 of $35(5.7 \%)$ \\
T3A + margins & 5 of $7(71.4 \%)$ & 5 of $11(45 \%)$ \\
T3B + margins & 0 of $0(0 \%)$ & 3 of $4(75 \%)$ \\
T4 + margins & 2 of $2(100 \%)$ & 0 of $0(0 \%)$ \\
Total positive margins & 22 of $50(44 \%)$ & 10 of $50(20 \%)$ \\
Surgeon 2 & & \\
Mean GG & 6.4 & 6.4 \\
Mean pre-op PSA & 8.9 & 6.4 \\
Mean prostate weight & 52.6 & 44.2 \\
pT2 (all) + margins & 8 of $38(27.5 \%)$ & 3 of $42(7 \%)$ \\
T3A + margins & 1 of $3(33.3 \%)$ & 2 of $3(67 \%)$ \\
T3B + margins & 4 of $9(44.4 \%)$ & 4 of $5(80 \%)$ \\
T4 + margins & NA & NA \\
Total positive margins & 13 of $50(26 \%)$ & 9 of $50(18 \%)$ \\
\hline
\end{tabular}

comers to our practice. To ensure that the open surgery and robotic surgery groups were clinically comparable, the pre-operative clinical parameters were attained and are presented in Table 1 . There were no significant differences between the groups with regard to age, pre-operative PSA or Gleason score. In addition, when stratified by risk profile there was no significant difference between the number of low-, intermediate-, and high-risk patients in each cohort (Table 1).

Table 2 compares the positive surgical margin rates in both groups of patients for both surgeons. The overall positive margin rate and $\mathrm{pT} 2$ positive margin rate for surgeon 1 dropped from 44 to $20 \%$ and from 37 to $5.7 \%$, respectively, after changing from open to robotic prostatectomy. For surgeon 2 , the overall positive margin rate decreased from 26 to $18 \%$ and the pT2 positive margin rate decreased from 27.5 to $7 \%$ after converting. A review of the locations for the positive margins in Table 3, demonstrates that the majority of positive margins for both surgeons in the open approach was at the apex, while for the robotic approach they were primarily at the bladder neck for one surgeon and posterolateral for the other.

Table 3 Location of the majority of positive margins of both surgeons by both approaches

\begin{tabular}{lll}
\hline Surgeon & Open & Robotic \\
\hline Surgeon 1 & Apex & Bladder neck \\
Surgeon 2 & Apex & Posterolateral \\
\hline
\end{tabular}

\section{Discussion}

Several studies have demonstrated that a positive surgical margin in a radical prostatectomy specimen is an independent predictor of disease recurrence, and the need to receive adjuvant therapy [17, 19, 21, 24-26, 34]. Therefore, prevention of positive surgical margins is a critical endpoint of radical prostatectomy. In a study performed by Eastham et al., it was demonstrated that the surgeon is an independent risk factor for positive surgical margins even after adjusting for various patient and disease characteristics. It was also found that surgeons with higher-volume practices have lower positive surgical margin rates [24]. Theoretically, the lower positive margin rates amongst high-volume surgeons is the result of technique refinement. The data has varied with regard to the impact of surgical technique on surgical margin status. A recent review of the open radical prostatectomy literature shows the incidence of positive surgical margins to ranges from 10 to $48 \%$ [24]. The positive margin rates for laparoscopic and robotic prostatectomy are relatively comparable and range between 5.7 and 29\% [35-38].

Several recent reports regarding surgical margin status in conjunction with the known advantages of robotic prostatectomy have demonstrated that expert open prostatectomists exhibit an improvement in their personal positive surgical margin rates after switching to the robotic approach [5, 7, 28-30,39]. Ahlering et al. has shown with the use of the da Vinci robot, surgeons can potentially benefit from the advantages of minimally invasive surgery and also be able to markedly reduce the risk of iatrogenic positive margins in patients with pT2 prostate cancer. This phenomenon has been replicated at several centers of excellence, but it is not known if this phenomenon applies to the private-practice urologist and, if so, what may be the key factors governing the robotic benefit. We believe that that the superior positive margin rates obtained after converting to the robotic-assisted approach can be explained by two main factors. The most critical factor was that the individual surgeons made a concerted effort to retrain in how to perform the procedure by observing and emulating experts. Second, the magnified three-dimensional (3-D) view of the pelvis, the precision movements afforded by the articulating robotic instrumentation and the bloodless field secondary to the pneumoperitoneum fostered a more precise dissection of the prostate. In combination, these two factors seemed to make a marked difference with regard to the ability of these two surgeons to obtain surgical margins.

Of critical importance to the successful improvement of margins was the seamlessness of the transition 
from open to robotic-assisted procedures. This was facilitated by several factors. First, both surgeons were proctored in their adoption of the robotic platform. The most critical factor was that the individual surgeons made a concerted effort to retrain in how to perform the procedure by observing and emulating experts. After attending a three-day training course at University of California, Irvine, consisting of observation and cadaver training in the laboratory, the two surgeons returned to their institutions and made a $100 \%$ conversion from open to robotic prostatectomy. Surgeon 1 had his first four cases proctored by an expert in robotic prostatectomy that acted as the bedside assistant for the cases. This greatly aided in acquiring proficiency at an early stage. Thereafter surgeon 1 was assisted by another robotic-trained but nonexpert attending urologist or a robotic-trained chief urology resident. Surgeon 2 also had 4 cases proctored by an expert robotic surgeon who acted as a bedside assistant but also had some additional training during the first 20 cases, during which an expert robotic surgeon acted as the bedside assistant while giving real-time feedback and input. Traditionally, the proctor for the first few robotic prostatectomies does not scrub in as an assistant; they give verbal instruction and advice. We feel that having a hands-on expert proctor as the assistant made a major difference with regard to achieving proficiency more quickly. In addition to aforementioned factors, the well-described advantages of the da Vinci robotic system (magnified 3-D view of the pelvis, the precision movements afforded by the articulating robotic instrumentation) as well as the bloodless field secondary to the pneumoperitoneum fostered a more precise dissection of the prostate. Secondly, a dedicated surgical staff was in place, including nurses and technologists all of whom underwent thorough training to familiarize them with the robot. And finally, the bedside surgical assistants were all comfortable with the robot, familiar with the surgery, and had themselves spent time at the console. With chief and senior residents at our institution logging roughly 50 robotic prostatectomies, and averaging 15 as primary surgeon, the bedside assistants were clearly quite facile with the entire procedure and greatly aided in making the transition as smooth as possible. All of these factors allowing this ease of transition were made possible by absolute dedication to the robotic platform. As was previously noted, since the decision to adopt the robot was made, only two case has been performed in the open manner. This has allowed full personnel and institutional commitment to the robotic approach. The wholehearted adoption allowed for rapid assimilation of the knowledge and skills, which are evidenced by the ability of these two surgeons to obtain negative surgical margins.

The open and robotic-assisted groups were similar with regard to preoperative characteristics and the transition from $100 \%$ open to $100 \%$ robotic prostatectomies was made abruptly in September of 2004 with no mixing of techniques. Therefore, we do not believe that the stated results can be explained by selection bias.

It might be argued that the perceived improvement in positive margin rates was attributable to the high number of positive margins in the open group. Although, the positive margin rates were high in the open group for both surgeons (36.6 and $27.5 \%$ ), they are still within the reported range noted in a current study [24]. Since most urologists do not routinely keep track of their margin data, we believe that these high rates may be reflective of many others. We feel strongly that the process of retraining and taking advantage of the robotic technology may help urologists improve their results markedly.

Finally, upon reviewing the locations for the positive margins, we learned that the majority of positive margins for both surgeons in the open approach was at the apex, indicating a likely problem with visualization of the deep pelvis, while during the robotic approach they were primarily at the bladder neck for one surgeon and posterolateral for the other. Since making this realization both surgeons have made concerted efforts to further refine their technique of dissection in these areas. Although not routinely performed during the study period, a video review of the cases with positive margins would have been helpful in this regard.

\section{Conclusion}

Changing from open to robotic-assisted radical prostatectomy may improve the ability of urologists to obtain negative surgical margins. This phenomenon does seem to apply to non-fellowship-trained urologists in private practice provided they undergo formal robotic training, and can be realized within the first 50 cases performed. Finally, it is only through the maintenance of a surgical database that stark realizations such as the results outlined in this paper can be realized.

\section{References}

1. Ahlering TE (2006) Robotic prostatectomy: is it the future? Urol Oncol 24:1-3

2. Eichel L, Ahlering TE, Clayman RV (2004) Role of robotics in laparoscopic urologic surgery. Urol Clin North Am 31:781-792 
3. Hemal AK, Menon M (2004) Robotics in urology. Curr Opin Urol 14:89-93

4. Kaul S, Menon M (2006) Robotic radical prostatectomy: evolution from conventional to VIP. World J Urol 24:152-160

5. Ahlering TE, Skarecky D, Lee D, Clayman RV (2003) Successful transfer of open surgical skills to a laparoscopic environment using a robotic interface: initial experience with laparoscopic radical prostatectomy. J Urol 170:1738-1741

6. Menon M, Tewari A, Peabody J (2003) Vattikuti Institute prostatectomy: technique. J Urol 169:2289-2292

7. Menon M, Hemal AK (2004) Vattikuti Institute prostatectomy: a technique of robotic radical prostatectomy: experience in more than 1000 cases. J Endourol 18:611-619

8. Tewari A, Peabody J, Sarle R et al (2002) Technique of da Vinci robot-assisted anatomic radical prostatectomy. Urology 60:569-572

9. Tewari A, Menon M (2003) Vattikuti Institute prostatectomy: surgical technique and current results. Curr Urol Rep 4:119-123

10. Tewari A, Kaul S, Menon M (2005) Robotic radical prostatectomy: a minimally invasive therapy for prostate cancer. Curr Urol Rep 6:45-48

11. Ahlering TE, Eichel L, Skarecky D (2005) Rapid communication: early potency outcomes with cautery-free neurovascular bundle preservation with robotic laparoscopic radical prostatectomy. J Endourol 19:715-718

12. Ahlering TE, Eichel L, Chou D, Skarecky DW (2005) Feasibility study for robotic radical prostatectomy cautery-free neurovascular bundle preservation. Urology 65:994-997

13. Ahlering TE, Skarecky D, Borin J (2006) Impact of cautery versus cautery-free preservation of neurovascular bundles on early return of potency. J Endourol 20:586-589

14. Menon M, Hemal AK, Tewari A, Shrivastava A, Bhandari A (2004) The technique of apical dissection of the prostate and urethrovesical anastomosis in robotic radical prostatectomy. BJU Int 93:715-719

15. Tewari A, Peabody JO, Fischer M et al (2003) An operative and anatomic study to help in nerve sparing during laparoscopic and robotic radical prostatectomy. Eur Urol 43:444454

16. Tewari A, Takenaka A, Mtui E et al (2006) The proximal neurovascular plate and the tri-zonal neural architecture around the prostate gland: importance in the athermal robotic technique of nerve-sparing prostatectomy. BJU Int 98:314323

17. Bianco FJ Jr, Riedel ER, Begg CB, Kattan MW, Scardino PT (2005) Variations among high volume surgeons in the rate of complications after radical prostatectomy: further evidence that technique matters. J Urol 173:2099-2103

18. Zincke H, Oesterling JE, Blute ML, Bergstralh EJ, Myers RP, Barrett DM (1994) Long-term (15 years) results after radical prostatectomy for clinically localized (stage T2c or lower) prostate cancer. J Urol 152:1850-1857

19. Bianco FJ Jr, Scardino PT, Eastham JA (2005) Radical prostatectomy: long-term cancer control and recovery of sexual and urinary function ("trifecta"). Urology 66:83-94

20. Hull GW, Rabbani F, Abbas F, Wheeler TM, Kattan MW, Scardino PT (2002) Cancer control with radical prostatectomy alone in 1,000 consecutive patients. J Urol 167:528-534

21. Blute ML, Bostwick DG, Bergstralh EJ et al (1997) Anatomic site-specific positive margins in organ-confined pros- tate cancer and its impact on outcome after radical prostatectomy. Urology 50:733-739

22. Catalona WJ, Smith DS (1994) 5-year tumor recurrence rates after anatomical radical retropubic prostatectomy for prostate cancer. J Urol 152:1837-1842

23. Chang SS, Cookson MS (2006) Impact of positive surgical margins after radical prostatectomy. Urology 68:249-252

24. Eastham JA, Kattan MW, Riedel E et al (2003) Variations among individual surgeons in the rate of positive surgical margins in radical prostatectomy specimens. J Urol 170:22922295

25. Karakiewicz PI, Eastham JA, Graefen M et al (2005) Prognostic impact of positive surgical margins in surgically treated prostate cancer: multi-institutional assessment of 5831 patients. Urology 66:1245-1250

26. Ohori M, Wheeler TM, Kattan MW, Goto Y, Scardino PT (1995) Prognostic significance of positive surgical margins in radical prostatectomy specimens. J Urol 154:1818-1824

27. Wieder JA, Soloway MS (1998) Incidence, etiology, location, prevention and treatment of positive surgical margins after radical prostatectomy for prostate cancer. J Urol 160:299-315

28. Ahlering TE, Eichel L, Edwards RA, Lee DI, Skarecky DW (2004) Robotic radical prostatectomy: a technique to reduce pT2 positive margins. Urology 64:1224-1228

29. Ahlering TE, Woo D, Eichel L, Lee DI, Edwards R, Skarecky DW (2004) Robot-assisted versus open radical prostatectomy: a comparison of one surgeon's outcomes. Urology 63:819-822

30. Menon M, Tewari A, Baize B, Guillonneau B, Vallancien G (2002) Prospective comparison of radical retropubic prostatectomy and robot-assisted anatomic prostatectomy: the Vattikuti Urology Institute experience. Urology 60:864-868

31. Tewari A, Srivasatava A, Menon M (2003) A prospective comparison of radical retropubic and robot-assisted prostatectomy: experience in one institution. BJU Int 92:205-210

32. Reiner WG, Walsh PC (1979) An anatomical approach to the surgical management of the dorsal vein and Santorini's plexus during radical retropubic surgery. J Urol 121:198-200

33. Walsh PC, Lepor H, Eggleston JC (1983) Radical prostatectomy with preservation of sexual function: anatomical and pathological considerations. Prostate 4:473-485

34. Epstein JI (1996) Incidence and significance of positive margins in radical prostatectomy specimens. Urol Clin North Am 23:651-663

35. Guillonneau B, el-Fettouh H, Baumert H et al (2003) Laparoscopic radical prostatectomy: oncological evaluation after 1,000 cases a Montsouris Institute. J Urol 169:1261-1266

36. Katz R, Salomon L, Hoznek A, de la TA, Antiphon P, Abbou CC (2003) Positive surgical margins in laparoscopic radical prostatectomy: the impact of apical dissection, bladder neck remodeling and nerve preservation. J Urol 169:2049-2052

37. Secin FP, Karanikolas N, Kuroiwa K, Vickers A, Touijer K, Guillonneau B (2005) Positive surgical margins and accessory pudendal artery preservation during laparoscopic radical prostatectomy. Eur Urol 48:786-792

38. Turk I, Deger S, Winkelmann B, Schonberger B, Loening SA (2001) Laparoscopic radical prostatectomy. Technical aspects and experience with 125 cases. Eur Urol 40:46-52

39. Patel VR, Tully AS, Holmes R, Lindsay J (2005) Robotic radical prostatectomy in the community setting - the learning curve and beyond: initial 200 cases. J Urol 174:269-272 